OPEN ACCESS

Edited by:

Arshan Nasir,

Los Alamos National Laboratory

(DOE), United States

Reviewed by:

Teiichiro Shiino,

National Center For Global Health

and Medicine, Japan

Thomas Leitner,

Los Alamos National Laboratory

(DOE), United States

*Correspondence:

Syed Hani Abid

m.haniabidi@gmail.com

tThese authors have contributed equally to this work and share first authorship

¥These authors have contributed equally to this work and share senior authorship

Specialty section:

This article was submitted to Virology,

a section of the journal

Frontiers in Microbiology

Received: 25 January 2021 Accepted: 21 July 2021 Published: 17 August 2021

Citation: Abidi SH, Nduva GM, Siddiqui D,

Rafaqat W, Mahmood SF Siddiqui AR, Nathwani AA, Hotwani A Shah SA, Memon S, Sheikh SA, Khan P, Esbjörnsson J, Ferrand RA and Mir F (2021) Phylogenetic and Drug-Resistance Analysis of HIV-1 Sequences From an Extensive Paediatric HIV-1 Outbreak in Larkana, Pakistan.

Front. Microbiol. 12:658186. doi: 10.3389/fmicb.2021.658186

\section{Phylogenetic and Drug-Resistance Analysis of HIV-1 Sequences From an Extensive Paediatric HIV-1 Outbreak in Larkana, Pakistan}

\author{
Syed Hani Abidi ${ }^{1 * t}$, George Makau Nduva ${ }^{2,3 t}$, Dilsha Siddiquilt, Wardah Rafaqat ${ }^{4}$, \\ Syed Faisal Mahmood ${ }^{5}$, Amna Rehana Siddiqui ${ }^{6}$, Apsara Ali Nathwani ${ }^{7}$, \\ Aneeta Hotwani ${ }^{7}$, Sharaf Ali Shah ${ }^{8}$, Sikander Memon ${ }^{9}$, Saqib Ali Sheikh ${ }^{9}$, \\ Palwasha Khan ${ }^{10}$, Joakim Esbjörnsson ${ }^{2,11 \neq}$, Rashida Abbas Ferrand $7,10 \neq$ and Fatima Mir" ${ }^{7 \neq}$ \\ ${ }^{1}$ Department of Biological and Biomedical Sciences, Aga Khan University, Karachi, Pakistan, ${ }^{2}$ Department of Translational \\ Medicine, Lund University, Lund, Sweden, ${ }^{3}$ Kenya Medical Research Institute-Wellcome Trust Research Programme, Kilifi, \\ Kenya, ${ }^{4}$ Medical College, Aga Khan University, Karachi, Pakistan, ${ }^{5}$ Department of Medicine, Aga Khan University, Karachi, \\ Pakistan, ${ }^{6}$ Department of Community Health Sciences, Aga Khan University, Karachi, Pakistan, ${ }^{7}$ Department of Pediatrics \\ and Child Health, Aga Khan University, Karachi, Pakistan, ${ }^{8}$ Bridge Consultants Foundation, Karachi, Pakistan, ${ }^{9}$ Sindh AIDS \\ Control Program, Ministry of Health, Karachi, Pakistan, ${ }^{10}$ Department of Clinical Research, London School of Hygiene \& \\ Tropical Medicine, London, United Kingdom, ${ }^{11}$ The Nuffield Department of Medicine, University of Oxford, Oxford, \\ United Kingdom
}

Introduction: In April 2019, an HIV-1 outbreak among children occurred in Larkana, Pakistan, affecting more than a thousand children. It was assumed that the outbreak originated from a single source, namely a doctor at a private health facility. In this study, we performed subtype distribution, phylogenetic and drug-resistance analysis of HIV-1 sequences from 2019 outbreak in Larkana, Pakistan.

Methods: A total of 401 blood samples were collected between April-June 2019, from children infected with HIV-1 aged 0-15 years recruited into a case-control study to investigate the risk factors for HIV-1 transmission. Partial HIV-1 pol sequences were generated from 344 blood plasma samples to determine HIV-1 subtype and drug resistance mutations (DRM). Maximum-likelihood phylogenetics based on outbreak and reference sequences was used to identify transmission clusters and assess the relationship between outbreak and key population sequences between and within the determined clusters. Bayesian analysis was employed to identify the time to the most recent common recent ancestor (tMRCA) of the main Pakistani clusters.

Results: The HIV-1 circulating recombinant form (CRF) 02_AG and subtype A1 were most common among the outbreak sequences. Of the treatment-naiive participants, the two most common mutations were RT: E138A (8\%) and RT: K219Q (8\%). Four supported clusters within the outbreak were identified, and the median tMRCAs of the Larkana outbreak sequences were estimated to 2016 for both the CRF02_AG and the subtype A1 clusters. Furthermore, outbreak sequences exhibited no phylogenetic mixing with sequences from other high-risk groups of Pakistan. 
Conclusion: The presence of multiple clusters indicated a multi-source outbreak, rather than a single source outbreak from a single health practitioner as previously suggested. The multiple introductions were likely a consequence of ongoing transmission within the high-risk groups of Larkana, and it is possible that the so-called Larkana strain was introduced into the general population through poor infection prevention control practices in healthcare settings. The study highlights the need to scale up HIV-1 prevention programmes among key population groups and improving infection prevention control in Pakistan.

Keywords: HIV-1, outbreak investigation, phylogenetic analysis, drug resistance, paediatric [MeSH]

\section{INTRODUCTION}

The HIV-1 pandemic has been established for 40 years and has resulted in approximately 32.7 million deaths worldwide (de Mendoza, 2019). One of the characteristic features of HIV-1 is its high mutation rate and recombination rate within and between hosts, leading to the emergence of distinct subtypes and circulating recombinant forms (CRFs) (Taylor et al., 2008; van Zyl et al., 2018). The subtypes can also recombine to give rise to unique recombinant forms (URFs) and minor HIV-1 variants (Taylor et al., 2008). The consequent genetic diversity that characterises HIV-1 infection has implications for virological control and transmission. Mutations encoded by the virus can interfere with epitope processing and recognition, leading to immune evasion. In addition, mutations may also lead to resistance to anti-retroviral drugs (van Zyl et al., 2018). Furthermore, certain immune- or drug-escape mutations may facilitate rapid transmission (van Zyl et al., 2018).

The phylogenetic and phylodynamic analysis of sequences derived from people living with HIV-1 (PLWH), particularly those who are part of an outbreak, can help answer fundamental questions such as the directionality and pattern of transmission and in understanding the introduction of HIV-1 into different regions, as well as identify clusters of transmission (Kosakovsky Pond et al., 2018; Dawson et al., 2020; Romero-Severson et al., 2020).

Pakistan has experienced a growing HIV-1 epidemic that is concentrated among three key population groups namely persons who inject drugs (PWID), transgender sex workers [also known as Hijra sex workers (HSW)], and men who have sex with men (MSM) (Raees et al., 2013) in whom prevalence is around 38-40, 11, and 7.5\%, respectively (Baqi et al., 1998; Waheed and Waheed, 2017; Hasan et al., 2018). Only 36,000 of an estimated 160,000 PLWH in Pakistan were aware of their HIV-1 positive status in 2020, and only 24,606 PLWH were receiving HIV-1 treatment of whom 7,693 were PWID (Esbjörnsson et al., 2016).

In April 2019, a cluster of fourteen HIV-1 diagnoses in children was reported in Ratodero, a town in Larkana district, Pakistan (Siddiqui et al., 2020). By December 2019, 1,167 children have been diagnosed with HIV-1 through a screening programme established in response to the outbreak (Brenner et al., 2007). Larkana has had three previous outbreaks of HIV, the first among PWID in 2003, the second in 2016 among 12 children in a paediatric hospital, and the third in 2016 among 56 individuals in a renal dialysis unit (Brenner et al., 2007; Altaf et al., 2016). These outbreaks were linked to poor infection prevention control practices including reuse of needles and inadequate blood screening.

For Pakistan, the outbreak in 2019 is unprecedented in terms of predominantly affecting children and its magnitude: prior to the outbreak, 1041 children had ever registered for HIV-1 care nationally over the past 13 years (Mir et al., 2020a). Early during the outbreak, media reports implicated a local doctor who had treated several of the infected children and who was later diagnosed with HIV, in spreading HIV-1 infection (Abi-Habib and Masood, 2019; Siddiqui et al., 2020).

In this study, we conducted a phylogenetic analysis to investigate the HIV-1 outbreak subtype and the pattern and source of transmission, and specifically whether this was a singlesource outbreak. Furthermore, we also analysed the sequences for presence of drug resistance mutations (DRMs).

\section{MATERIALS AND METHODS}

\section{Study Design and Setting}

This study was embedded in an individually matched casecontrol study that recruited 401 cases defined as children aged $0-15$ years who registered for HIV-1 care at the Paediatric Treatment Center at Shaikh Zayed Children's Hospital (Siddiqui et al., 2020). This centre was established by the Sindh AIDS Control Program in response to the outbreak. Prior to the outbreak, the nearest paediatric HIV-1 services were in the provincial capital, Karachi, situated more than 400 kilometres from Ratodero. Age-, sex-, and neighbourhoodmatched HIV-uninfected controls were also recruited. An interviewer-administered questionnaire collected data on risk factors for HIV-1 infection. A blood sample was collected for Hepatitis B and C serology, and for HIV-1 phylogenetic studies (in cases only). Written informed consent was obtained from guardians and assent from participants. The study was approved by the Aga Khan University Ethical Review Committee (ERC\# 2019-1536-4200). Prior to sample collection, written informed consent was obtained from the guardians, and if the child was able to understand the study procedures, a written assent was obtained. The study objectives were explained to the patient at the time of taking consent/assent and patients were informed that their identities will remain confidential. The 
participants were also informed that they had the opportunity to withdraw from the study at any given time and that this would have no consequences on the treatment or the care that they would receive.

\section{DNA Amplification and HIV-1 Genotyping}

Proviral DNA was extracted from blood samples obtained from cases using Qiagen's QIAamp DNA blood mini kit according to the manufacturer's instructions and stored at $-80^{\circ} \mathrm{C}$. The pol gene was amplified from each extracted DNA sample using a two-step nested polymerase chain reaction (PCR) strategy. Two sets of outer primers were used: Forward (POLOF CAGCATGYCAGGGAGTRGGRGGACC, amino acid; 1832-1856, HXB2, IBF1 5'-AAATGATGACAGCATGTCAG GGAGT-3'. nt 1823-1847, HXB2) and Reverse (IBR1 5'-AACTT CTGTATATCATTGACAGTCCA-3'. nt 3303-3328, HXB2). The first-round product was used as a template for the second round with primer set, Forward (POLIF 5'-AGGCTAATTTT TTAGGGAARATYTGGCCTTCC-3'. nt 2078-2109, amino acid PR: 1-9; HXB2) and reverse (RTOUT3 5'-TATGTCATT GACAGTCCAGCT-3'. nt 3300-3320, amino acid RT: 251-257 HXB2) (Tariq et al., 2018). PCR Mastermix (ABM) Bestaq (2X) cat\# G464 and Hotstart (2X) cat\# G906 were used to prepare a $25 \mathrm{ul}$ reaction mixture, and $0.8 \mathrm{pmol}$ and $0.6 \mathrm{pmol}$ primer used for the first and second round, respectively. Thermo cycle conditions were as follows: denaturation at $95^{\circ} \mathrm{C}$ for $5 \mathrm{~min}$, followed by 40 cycles of denaturation at $95^{\circ} \mathrm{C}$ for $1 \mathrm{~min}$, annealing at $50^{\circ} \mathrm{C}$ for IBF $1 / \mathrm{IBR} 1$ and $55^{\circ} \mathrm{C}$ for POLOF/IBR 1 sets (round 1 ), $60^{\circ} \mathrm{C}$ (round 2) for $20 \mathrm{~s}$, extension at $72^{\circ} \mathrm{C}$ for $1 \mathrm{~min}$ with a final extension of at $72^{\circ} \mathrm{C}$ for $7 \mathrm{~min}$. The protocol was run with positive and negative control to confirm results. The amplicons underwent sequencing using the Sanger sequencing platform (Macrogen, South Korea) and the sequences were deposited in the GenBank and assigned the accession numbers MN698251-MN698253, MN698255-MN698264, MN752136, MN752137, and MT748850-MT749178.

\section{Subtype Analysis}

HIV-1 pol sequence data used in the study comprised of either newly generated sequences (referred to as outbreak sequences) or Pakistani HIV-1 pol sequences retrieved from the Los Alamos HIV sequence database ${ }^{1}$ (referred to as published Pakistani sequences representing Pakistani people who inject drugs (PWID), heterosexuals, sex workers, and other individuals with unknown transmission risk) (Kuiken et al., 2003). Outbreak sequences and published Pakistani HIV-1 pol sequences (referred to as the Pakistani dataset) were aligned with HIV-1 Group M (subtypes A-K + Recombinants) subtype reference sequences ${ }^{1}$ using the MAFFT algorithm in Geneious Prime 2019 (Larkin et al., 2007). Subtyping of each sequence was determined by maximum-likelihood (ML) phylogenetic analysis in PhyML using the general time-reversible substitution model with a gamma-distributed rate variation and proportion of invariant sites $($ GTR $+\Gamma 4+\mathrm{I}$ ) (Guindon et al., 2010). Branch support was estimated using the approximate likelihood ratio test

${ }^{1}$ http://www.hiv.lanl.gov with the Shimodaira-Hasegawa-like procedure (SH-aLRT) in PhyML, where SH-aLRT support values $\geq 0.90$ were considered significant (Guindon et al., 2010). Phylogenies were visualised in FigTree v1.4.4. ${ }^{2}$

\section{Cluster Analysis}

To identify local transmission clusters, subtype-specific ML phylogenies were reconstructed for the main/predominant HIV-1 strains identified in the outbreak. The most similar nonPakistani sequences for each sequence in the Pakistani dataset were retrieved from the NCBI GenBank and used as reference sequences as previously described (Esbjörnsson et al., 2016; Nazziwa et al., 2020; Nduva et al., 2020). The Pakistani dataset and GenBank reference sequences were aligned by subtype or CRF and subtype/CRF-specific phylogenies were reconstructed in PhyML (Guindon et al., 2010). Monophyletic clusters having aLRT-SH $\geq 0.90$ and comprising $\geq 80 \%$ Pakistani sequences were defined as Pakistani-specific clusters (Esbjörnsson et al., 2016; Hassan et al., 2017; Nazziwa et al., 2020; Nduva et al., 2020). Clusters were classified into dyads (2 sequences), networks (3-14 sequences), or large clusters (>14 sequences) (Esbjörnsson et al., 2016).

\section{Estimating Dates of the Most Recent Common Ancestor for Each Cluster}

The dates of origin (time to the most recent common ancestor; tMRCA) of the large Pakistani-specific HIV-1 clusters were estimated using Bayesian Markov Chain Monte Carlo (MCMC) inference in BEAST (v1.10.4) (Gill et al., 2020). All Larkana outbreak sequences were sampled in 2019 and did not independently have a sufficient temporal signal for inference of the dates of origin. Hence, supplementary non-Pakistani reference sequences (seven CRF02_AG and six sub-subtype A1 sequences), and Pakistani sequences from previous outbreaks (33 CRF02_AG and 11 sub-subtype A1 sequences) sampled from different years were used to inform the temporal signal (assessed in TempEst v1.5.3) (Rambaut et al., 2016). Subtypespecific Bayesian inferences were done in BEAST 1.10.4 using the Bayesian Skygrid model with an uncorrelated lognormal relaxed clock and inferred under the GTR + $44+$ I substitution model (Drummond et al., 2005; Baele et al., 2012; Gill et al., 2013; Suchard et al., 2018). BEAST runs of 500 million generations were performed, sampling every 50,000th iteration, and discarding the first $10 \%$ of samples as burn-in. Convergence was determined in Tracer v.1.7.0, defined as effective sample sizes (ESS) $\geq 200$ (Suchard et al., 2018). Maximum clade credibility (MCC) trees were summarised in Tree-Annotator v1.10.4 and visualised in Figtree (v1.4.4).

\section{Drug Resistance Mutation Analysis}

Mutations in the HIV-1 pol gene (protease and reverse transcriptase region) associated with resistance against protease and reverse transcriptase inhibitors was determined using the Stanford HIV-1 drug resistance database (Rhee et al., 2003),

\footnotetext{
${ }^{2}$ https://github.com/rambaut/figtree/releases
} 
and confirmed using the 2019 Update of the Drug Resistance Mutations in HIV-1 by the International AIDS SocietyUnited States (Wensing et al., 2019). The DRMs were also classified as those conferring high, intermediate, low, and potential low-level resistance using the algorithm described in Stanford HIV-1 drug resistance database and IAS-United States report.

\section{RESULTS}

\section{Study Population}

Of the blood samples obtained from the 401 cases enrolled in the case-control study, 344 were successfully amplified and sequenced. The remaining 57 samples failed to amplify possibly due to low viral load secondary to receiving antiretroviral treatment or due to genomic diversity attributed to quasispecies in an individual (Debyser et al., 1998; Gupta et al., 2017). Out of 344 cases, socio-demographic information was available for 321 sequences, while information for 23 cases was missing. The median age of participants was three (IQR: 2-5) years and $65 \%$ were male (Table 1$)$. The majority $(84.4 \%)$ were taking ART at the time of sampling for a median period of 41 days (range: 22-192 days). The ART regimen comprised of zidovudine, lamivudine, and nevirapine. Most participants lived

TABLE 1 | Characteristics of study participants.

\begin{tabular}{ll}
\hline Category/variable & Total no. (\%) \\
\hline Age (years) & \\
$0-5$ & $248(77 \%)$ \\
$5-10$ & $58(18 \%)$ \\
$10-15$ & $15(5 \%)$ \\
Sex & \\
Male & $208(65 \%)$ \\
Female & $113(35 \%)$ \\
Location & \\
Ratodero, Larkana & $132(41 \%)$ \\
Outside Ratodero but within Larkana district & $141(44 \%)$ \\
Shikarpur district & $44(13.7 \%)$ \\
Jafarabad district & $1(0.3 \%)$ \\
Khairpur district & $1(0.3 \%)$ \\
Nawabshah district & $1(0.3 \%)$ \\
ART History & \\
Naïve & $50(15.6 \%)$ \\
Experienced & $271(84.4 \%)$ \\
ART duration (268) & \\
$<30$ days & $93(28.97 \%)$ \\
$>30-180$ days & $175(54.5 \%)$ \\
HCVand HBV co-infections & \\
HBV positive & $75(23.4 \%)$ \\
HCV positive & $26(8 \%)$ \\
Maternal HIV-1 status & \\
HIV-1 positive mother & $28(8.7 \%)$ \\
Mother's HIV-1 status unknown & $4(1.2 \%)$ \\
\hline &
\end{tabular}

in Ratodero, the epicentre of the outbreak, while the remainder were from other areas of Larkana district and neighbouring districts (Table 1).

\section{Pakistani HIV-1 Sequence Dataset and HIV-1 Subtypes and CRFs}

Overall, we analysed $532 \mathrm{HIV}-1$ partial pol sequences in the Pakistani dataset including outbreak sequences $(N=344)$ and previously published sequences $(N=188)$. HIV-1 CRF02_AG $(N=338,63.5 \%)$ dominated the outbreak, followed by subsubtype A1 $(N=149,28.0 \%)$. Additional subtypes found in the outbreak were subtype $\mathrm{C}(N=20,3.8 \%)$, subtype $\mathrm{G}(N=8,1.5 \%)$, CRF35_AD $(N=7,1.3 \%)$, subtype B $(N=5,0.9 \%)$, and subtype $\mathrm{D}(N=5,0.9 \%$, Figure 1$)$.

\section{IDENTIFICATION OF PAKISTANI-SPECIFIC HIV-1 TRANSMISSION CLUSTERS}

ML phylogenies were reconstructed independently for CRF02_AG $(N=338)$ and sub-subtypes A1 $(N=149)$, which were the most prevalent HIV-1 strains in the outbreak. The final reference dataset comprised of 310 non-Pakistani reference sequences for CRF02_AG, and 382 for sub-subtype A1 remained. Overall, 291 (86.1\% CRF02_AG outbreak sequences) and 59 (40.0\% sub-subtype A1 outbreak sequences) sequences formed 17 supported Pakistani clusters (size range: 2-283 sequences per cluster). These 17 clusters included 9 dyads (52.9\% of all clusters), six networks (33.3\%), and two large clusters $(11.8 \%$, Table 2 and Figures 2A,B). Sub-subtype A1 clusters were more common $(N=13,76.5 \%)$ as compared to the CRF02_AG clusters $(N=4,23.5 \%)$. Of the 344 outbreak sequences, $312(90.7 \%)$ were found in four distinct clusters. More specifically, 283 sequences were found in one large CRF02_AG cluster (Figure 2A), two sequences in a CRF02_AG dyad (Figure 2A), 22 sequences in one large sub-subtype A1 cluster (Figure 2B), and four sequences in a sub-subtype A1 network (Figure 2B). HIV-1 clusters of outbreak sequences showed no evidence of mixing with other HIV-1 risk groups of Pakistan. Instead, the Pakistani sequences that were not part of the outbreak formed an exclusive Pakistani cluster of non-outbreak sequences (Table 2).

\section{Estimation of Time to the Most Recent Common Ancestor (tMRCA)}

The tMRCAs were estimated for the two large clusters (one CRF02_AG and one subtype A1 cluster) comprising of the outbreak sequences. The median tMRCA of the CRF02_AG cluster was estimated to 2016 (95\% higher posterior density [HPD] interval: 2015-2017), and the tMRCA of the subsubtype A1 cluster was estimated to be 2016 (95\% HPD interval: 2015-2018). In addition, the divergence time between the outbreak sequences and that of other high-risk groups in Pakistan, such as PWID, was dated to the year 1999 (95\% HPD interval: 1994-2010) for the CRF02_AG cluster, 


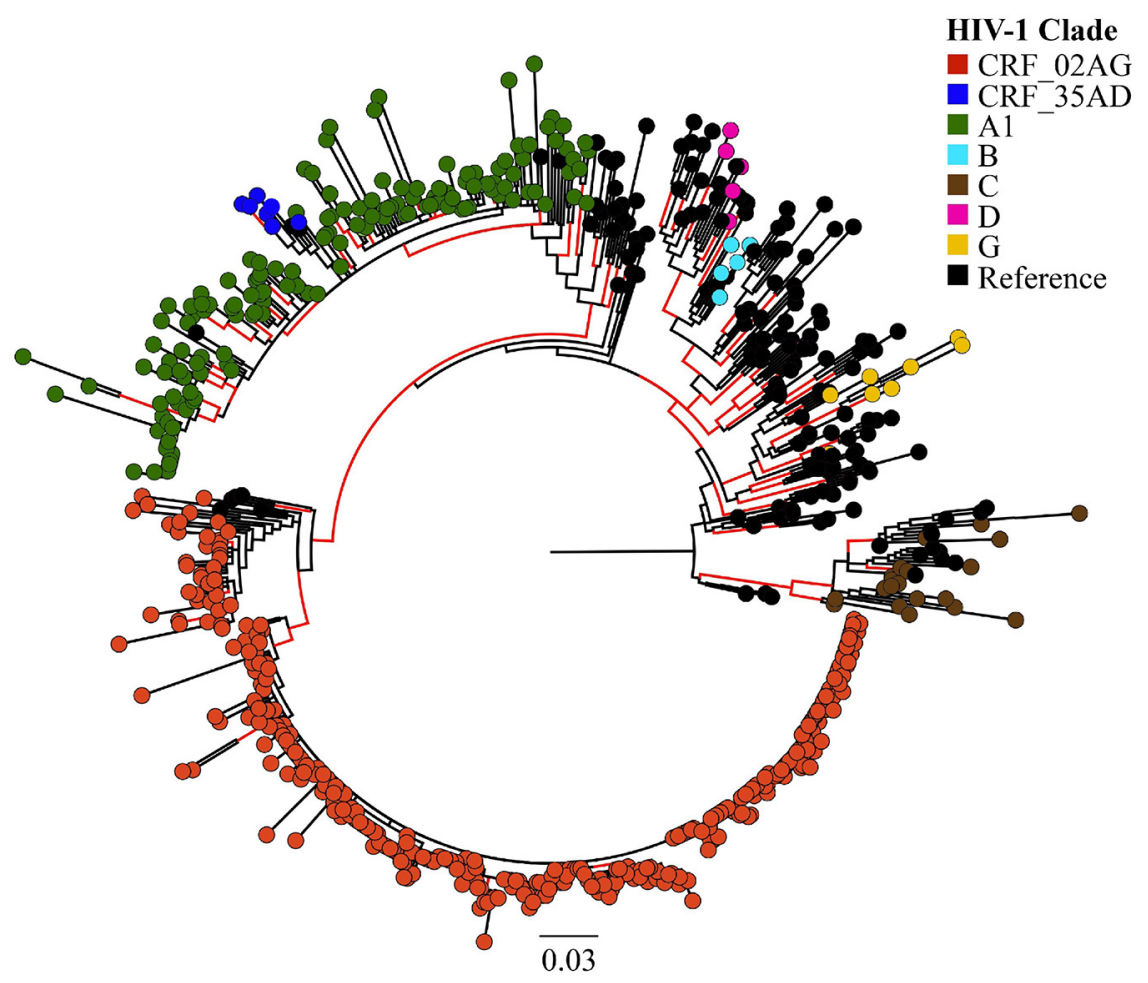

FIGURE 1 | Maximum likelihood (ML)-based subtyping of HIV-1 sequences: ML-based phylogeny/subtyping of 532 HIV-1 sequences (including 344 outbreak sequences from Larkana-Pakistan). Branch tips on the phylogenetic tree are coloured according to HIV CRF/subtype (Red: CRF_02AG; Blue: CRF_35AD, Green: subtype A1; Sky Blue: subtype B; Brown: subtype C; Pink: subtype D; Yellow: subtype G; Black: reference sequences from the Los Alamos HIV database). Scale bar units are nucleotide substitutions per site.

TABLE 2 | The number of Pakistani transmission clusters by cluster size, risk group, and shared drug resistance mutations.

\begin{tabular}{lccll}
\hline Cluster name & HIV-1 subtype & Number of Tips & Transmission group & Number with shared DRM \\
\hline Cluster_A1_1 & A1 & 22 & Paediatric & RT:E138A; $N=21$ \\
Cluster_A1_2 & A1 & 3 & Paediatric & RT:E138A; $N=3$ \\
Cluster_A1_3 & A1 & 2 & Unknown & \\
Cluster_A1_4 & A1 & 2 & MSM/unknown & \\
Cluster_A1_6 & A1 & 2 & PWID & \\
Cluster_A1_7 & A1 & 2 & Unknown & \\
Cluster_A1_8 & A1 & 2 & Unknown & \\
Cluster_A1_9 & A1 & 2 & Unknown & \\
Cluster_A1_10 & A1 & 9 & PWID/unknown & \\
Cluster_A1_11 & A1 & 2 & Unknown & \\
Cluster_A1_12 & A1 & 5 & Unknown & MSM/unknown \\
Cluster_A1_15 & A1 & 3 & CWSW/unknown & RT:K219Q; $N=5, R T: K 103 N ; N=19$, \\
Cluster_A1_16 & A1 & 3 & Paediatric & \\
Cluster_CRF02AG_1 & CRF02_AG & 283 & RT:E138A; $N=7, R T: V 17 ; N=10$ \\
Cluster_CRF02AG_2 & CRF02_AG & 4 & RT:E138A; $N=1, R T: V 17 ; N=2$ \\
Cluster_CRF02AG_3 & CRF02_AG & 2 & Paediatric \\
Cluster_CRF02AG_4 & CRF02_AG & 2 & Unknown &
\end{tabular}

Abbreviations: MSM, men who have sex with men; PWID, people who inject drugs; CWSW, female sex worker; Unknown, sequences lacking information on risk group or transmission route; Outbreak, sequences collected from the 2019 Larkana outbreak; RT, HIV-1 reverse transcriptase gene. 

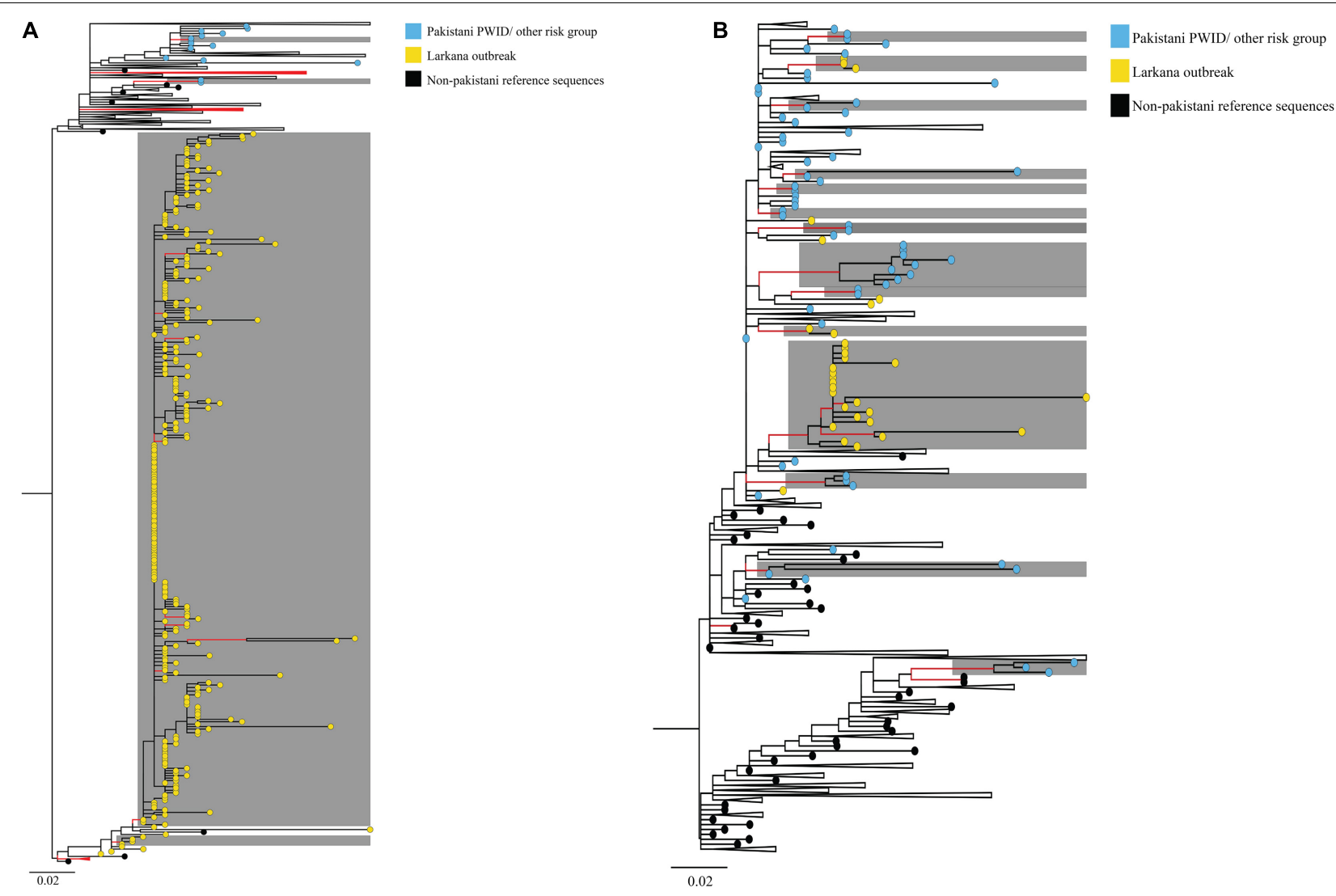

FIGURE 2 | Maximum-likelihood trees summarising clustering patterns of different risk groups in Pakistani: Trees represent (A) CRF02_AG, and (B) sub-subtype A1 transmission clusters, respectively. Each phylogeny is rooted at the midpoint, and branches are arranged in increasing node order. Branches with aLRT-SH support $\geq 0.9$ are coloured red. Monophyletic clusters with aLRT-SH support $\geq 0.9$ and which have $\geq 80 \%$ sequences from Pakistan are highlighted in grey. To enhance cluster visualisation, some branches containing either reference sequences or Pakistani sequences that are not part of clusters have been collapsed (shown as black triangles, with the recent end of the triangle indicating the latest sampling date). Branch tips within respective clusters are coloured as per cluster risk group (Bluishgreen: MSM; Sky blue: Pakistani PWID/other risk groups IDU; Yellow: Larkana paediatric sequences; and Black: Non-Pakistani Reference sequences). Scale bars represent the genetic distance in substitutions per site in both phylogenies. As an overview, among CRF02_AG sequences, whereas PWID and other risk groups formed small clusters (size range 2-4 sequences per cluster), paediatric sequences from the Larkana outbreak formed one large cluster ( $N=283$ sequences). Likewise, among subtype A1 sequences, PWID and individuals from other risk groups formed several small clusters (size range, 2-9 sequences per cluster), whilst paediatric sequences from the Larkana outbreak formed one large cluster ( $N=22$ sequences).

and 2004 (95\% HPD interval: 1998-2014) for sub-subtype A1 cluster (Figure 3).

\section{Drug Resistance Mutation Analysis}

Drug resistance mutation analysis was conducted on both ARTnaïve (15.6\%) and ART- experienced (84.4\%) sequences from the outbreak. Among ART naïve individuals, 15 (30\%) had drug resistance mutations (DRM); the most common of which were the Reverse Transcriptase (RT):E138A (8.0\%) and RT:K219Q (8.0\%) mutations. Among treatment-experienced individuals, the most common mutations were RT:E138A (12.92\%), RT:K219Q (8.86\%), and RT:K103N (6.64\%). The DRMs RT:E138A and RT:K103N confer resistance against non-nucleoside reverse transcriptase inhibitors (NNRTI) rilpivirine and efavirenz, respectively, while RT:K219Q is associated with resistance against nucleoside reverse transcriptase inhibitors (NRTI) zidovudine. Similarly, DRM PI:N88D, associated with resistance against protease inhibitors (PI), such as atazanavir/ritonavir, and tipranavir/ritonavir was observed in two treatmentexperienced participants, while DRMs PI:M46L, PI:D30N, PI:N83D, PI:K43T, PI:G73S, PI:L33F were seen in one treatmentexperienced individual each (Table 3). No DRM against protease inhibitors was observed in ART-naïve individuals. Analysis also showed that 114 (42\%) patients with DRMs belonged to the drug-experienced group, while, 15 (30\%) belonged to the ART-naïve group (Table 3).

Next, sequences with any DRMs were analysed for clustering. Among the 78 sequences from the Larkana outbreak with prevalent drug resistance mutations, the DRM RT:K219Q was shared between five sequences whereof all clustered together in cluster_CRF02_AG_1 (Tables 2,3). The DRM RT:M184V was found in two sequences - both in cluster_CRF02_AG_1. The DRM RT:K103N was found among 21 sequences, whereof 19 sequences were found in cluster (cluster_CRF02_AG_1). The two remaining sequences were not part of any cluster. The DRM RT:E138A was found in 38 sequences of different subtypes 


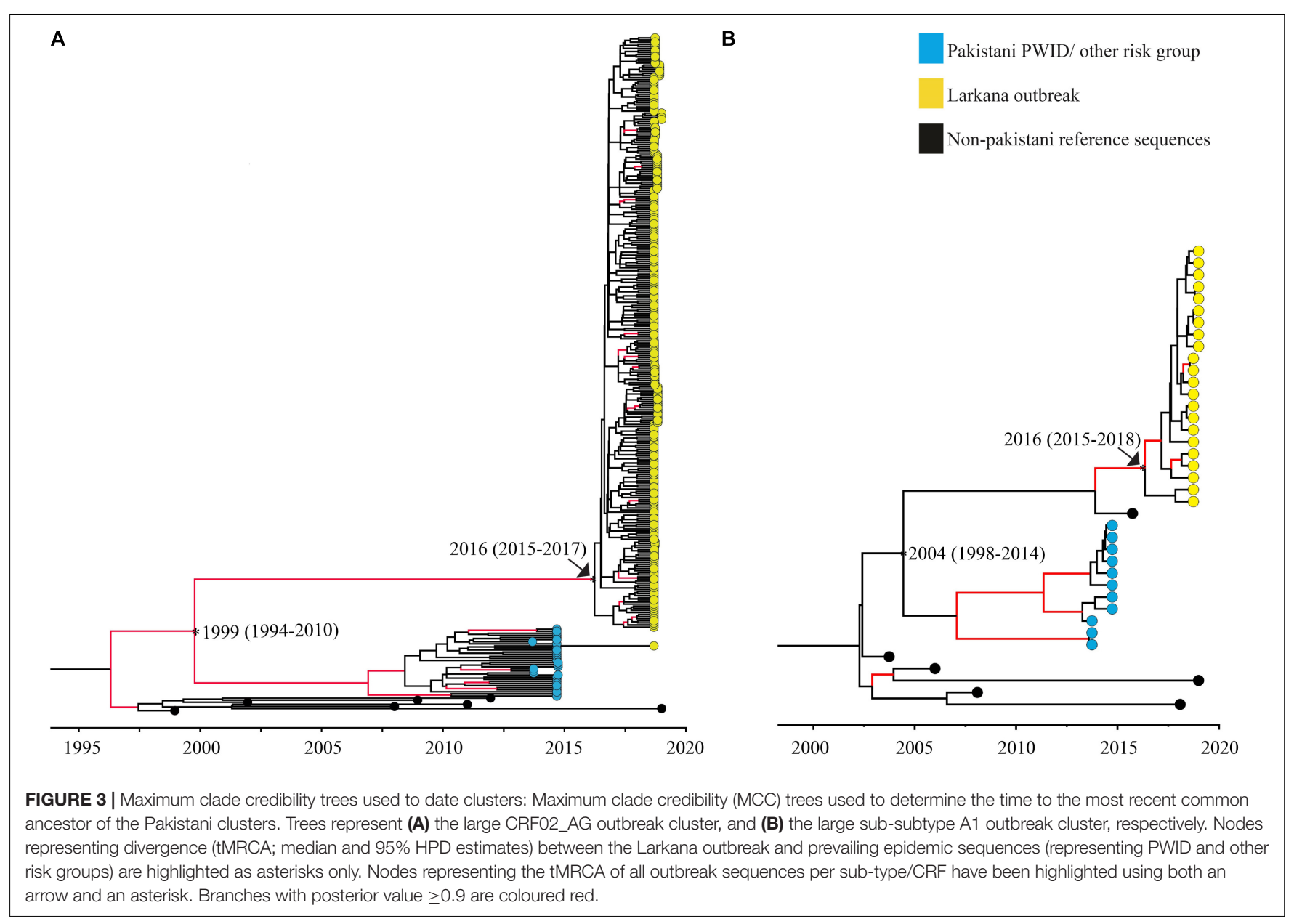

including 21 sequences in cluster_A1_1, three sequences in cluster A1_2, seven sequences in cluster_CRF02_AG_1, one sequence in cluster_CRF02AG_2, and six sequences that were not part of a cluster. The DRM RT:V179L was distributed among 10 sequences in cluster_CRF02AG_1, and two sequences in cluster_CRF02AG_2 (Tables 2,3).

\section{DISCUSSION}

In this study, we determined the HIV-1 subtype distribution, phylodynamics, and presence of HIV-1 drug-resistance mutations of the 2019 HIV-1 outbreak among children in Larkana, Pakistan. Seventeen distinct clusters were found among the Larkana outbreak sequences, indicating that HIV-1 was introduced from multiple sources rather than from a single source, as previously suggested (Arif, 2019; Siddiqui et al., 2020). A similar large-scale nosocomial HIV-1 outbreak was reported in Libya 1998-1999 (Visco-Comandini et al., 2002), where a monophyletic HIV-1 CRF02_AG cluster was identified among children visiting the El-Fatih Children's hospital in Benghazi. The HIV-1 transmission in the Libyan children was suggested to originate from contaminated intravenous injections (although not from blood or blood products) (Visco-Comandini et al.,
2002). Similarly, the HIV-1 transmissions in the 2019 Larkana outbreak were strongly associated with visits to both public and private sector facilities, but not with a single healthcare facility, and with receipt of infusions, injections and blood transfusions (Mir et al., 2020b), implying transmission through poor infection control practices. Some of the children had HIV-1 positive mothers, raising the possibility of mother-tochild HIV-1 transmission. However, the possibility of vertical transmission could not be verified due to unavailability of maternal samples. Taken together, our results indicate that the Larkana outbreak was not the result of a single-source transmission from one health care practitioner, but may have resulted from through multiple sources at different health facilities. Moreover, and as previously suggested, our results indicate that poor infection prevention control is still present in Larkana (Altaf, 2018).

The HIV-1 subtype analysis showed a high prevalence of CRF02_AG and sub-subtype A1. This is consistent with previous reports indicating sub-subtype $\mathrm{A} 1$ as the dominant circulating subtype in Pakistan (Khan et al., 2018), whereas CRF02_AG has shown increasing prevalence more recently (Chen et al., 2016). A study reporting on the molecular epidemiology of HIV-1 in Pakistan suggested that sub-subtype A1 was introduced in Pakistan 1989 (95\% HPD: 1984-1994) (Chen et al., 2016). 
TABLE 3 | Classification of the Drug resistance mutations.

\begin{tabular}{|c|c|c|c|c|c|c|}
\hline \multicolumn{2}{|c|}{ Drug } & \multirow{2}{*}{$\begin{array}{l}\text { Mutation } \\
\mathrm{K} 219 \mathrm{Q}\end{array}$} & \multirow{2}{*}{$\begin{array}{c}\begin{array}{c}\text { Naïve, } \boldsymbol{N}(\%) \\
(\boldsymbol{n}=\mathbf{5 0 )}\end{array} \\
4(8.00)\end{array}$} & \multirow{2}{*}{$\begin{array}{l}\begin{array}{c}\text { Experienced, } \boldsymbol{N}(\%) \\
(\boldsymbol{n}=\mathbf{2 7 1})\end{array} \\
1(0.37)\end{array}$} & \multirow{2}{*}{$\begin{array}{c}\begin{array}{c}\text { Mutation } \\
\text { classification }\end{array} \\
\text { Major }\end{array}$} & \multirow{2}{*}{$\begin{array}{l}\text { Drug associated } \\
\text { with resistance }\end{array}$} \\
\hline \multirow{5}{*}{ 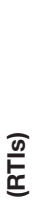 } & & & & & & \\
\hline & \multirow{4}{*}{$\begin{array}{l}\frac{\infty}{5} \\
\frac{1}{z}\end{array}$} & M184V & $0(0.00)$ & $2(0.74)$ & Major & ABC, 3TC \\
\hline & & M184I & $0(0.00)$ & $1(0.37)$ & Major & ABC, 3TC \\
\hline & & L210W & $0(0.00)$ & $1(0.37)$ & Major & $\mathrm{AZT}$ \\
\hline & & K70R & $0(0.00)$ & $2(0.74)$ & Major & $\mathrm{ABC}, \mathrm{TDF}, \mathrm{AZT}$ \\
\hline \multirow{7}{*}{ 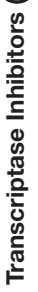 } & & Y115F & $0(0.00)$ & $1(0.37)$ & Major & TDF, ABC \\
\hline & & A98G & $0(0.00)$ & $3(1.11)$ & Minor & NVP, EFV \\
\hline & & $\mathrm{K} 103 \mathrm{~N}$ & $2(4.00)$ & $19(7.0)$ & Major & EFV, NVP \\
\hline & & K101E & $0(0.00)$ & $1(0.37)$ & Major/Minor & NVP, EFV \\
\hline & & E138A & $4(8.00)$ & $35(12.92)$ & Major/Minor & ETR, RPV \\
\hline & & E138K & $1(2.00)$ & $0(0)$ & Major/Minor & EFV, NVP \\
\hline & & E138G & $0(0.00)$ & $1(0.37)$ & Major/Minor & EFV, NVP \\
\hline \multirow{6}{*}{ 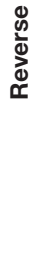 } & $\stackrel{\underline{0}}{\underline{n}}$ & V179L & $2(4.00)$ & $10(3.69)$ & Major & EFV, NVP \\
\hline & z & V179F & $1(2.00)$ & $0(0)$ & Minor & EFV, NVP \\
\hline & & Y181C & $0(0.00)$ & $1(0.37)$ & Major & EFV, NVP \\
\hline & & G190A & $0(0.00)$ & $2(0.74)$ & Major/Minor & EFV, NVP \\
\hline & & V106I & $1(2.00)$ & $2(0.74)$ & Minor & NVP \\
\hline & & F227C & $0(0.00)$ & $1(0.37)$ & Major/Minor & EFV, NVP \\
\hline \multirow{8}{*}{ 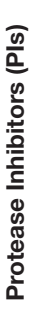 } & & L234I & $0(0.00)$ & $1(0.37)$ & Minor & DOR \\
\hline & & H221Y & $0(0.00)$ & $1(0.37)$ & Major & EFV, NVP \\
\hline & & M46L & $0(0.00)$ & $1(0.37)$ & Minor/Major & $\mathrm{LPV} / \mathrm{r}$ \\
\hline & 흠 & D30N & $0(0.00)$ & $1(0.37)$ & Major & NFV \\
\hline & $\Sigma$ & N88D & $0(0.00)$ & $2(0.74)$ & Minor & ATV/r, SQV/r, NFV \\
\hline & & N83D & $0(0.00)$ & $1(0.37)$ & Minor/Major & ATV/r, TPV/r \\
\hline & 을 & G73S & $0(0.00)$ & $1(0.37)$ & Minor & $\mathrm{LPV} / \mathrm{r}$ \\
\hline & $\Sigma$ & L33F & $0(0.00)$ & $1(0.37)$ & Minor & $\mathrm{LPV} / \mathrm{r}$ \\
\hline
\end{tabular}

The mutation classification column, indicates mutations that are classified as major or minor DRMs.

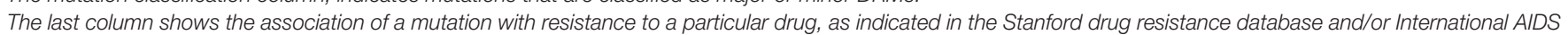
Society (IAS) 2019 report, respectively, while bold drug names indicate the major resistance by IAS 2019.

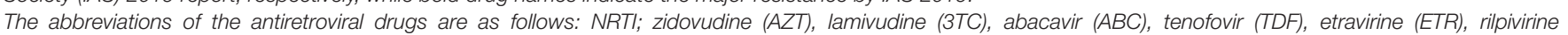

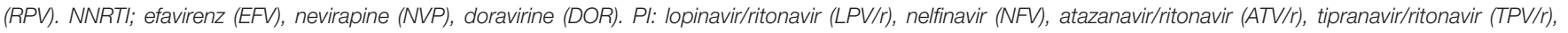
Saquinavir/ritonavir (SQV/r). \% = percentage of total participants.

After this introduction, sub-subtype A1 disseminated rapidly to become the dominant HIV-1 strain (Chen et al., 2016). However, no information exists about the introduction of CRF02_AG in Pakistan, although it has been shown that the prevalence of HIV-1 CRF02_AG infections are increasing - especially in highrisk populations (Cholette et al., 2020). It is therefore possible that HIV-1 CRF02_AG become the dominant HIV-1 strain in Pakistan over time.

In our analysis, the tMRCA for the two large clusters were both estimated to 2016 (CRF02_AG: 95\% HPD interval: 2015-2017; A1: 95\% HPD interval: 2015-2018), suggesting ongoing HIV-1 transmissions several years prior to the 2019 outbreak. Interestingly, the estimated tMRCA of the two main clusters coincides with the time of the previously reported HIV-1 outbreak in Larkana in 2016, which also occurred in a nosocomial setting (Brenner et al., 2007; Siddiqui et al., 2020). Furthermore, the majority $(80 \%)$ of children identified in the outbreak had stage 3 or 4 disease (the moderately and severely symptomatic stage, respectively, mostly associated with chronic to acute-chronic infection) (Weinberg and Kovarik, 2010; Altaf et al., 2016), indicating that some of the HIV-1 infections identified in the 2019 outbreak occurred a few years prior to 2019. Moreover, no reference sequence from the global HIV-1 epidemic was found in the Pakistani clusters, suggesting a localised HIV-1 epidemic. It is possible that the transmission may have been ongoing within healthcare settings, and the active screening programme implemented by the provincial AIDS Control Program in response to the initial diagnosis of HIV-1 in 14 children in 2019 identified these infections.

Phylogenetic analyses demonstrated no mixing between the outbreak sequences and sequences previously obtained from other high-risk groups in Pakistan. This was further supported by dating of the outbreak and other Pakistani clusters, indicating that the splitting time points between the outbreak sequences and that of other high-risk groups in Pakistan occurred between 1994 and 2012 (combined HPD interval). This suggests that HIV-1 most likely were introduced in Larkana between 10 and 27 years ago, and that the HIV-1 transmissions are now localised to certain regions of Larkana. It is possible that HIV-1 arose from nosocomial routes through 
the widespread poor infection prevention control practices in healthcare settings and contaminated blood (Cotton and Rabie, 2020; Mir et al., 2020a).

DRM analysis showed the presence of multiple DRMs associated with resistance against reverse transcriptase inhibitors, some of which were shared among sequences in the identified clusters. The presence of shared DRMs may indicate transmission of drug-resistant strains in outbreak sequences. The most prevalence DRM identified among both ART-experienced and ART-naïve individuals was RT:E138A which confers a high-level resistance to NNRTIs such as Rilpivirine (Jeulin et al., 2014). A high prevalence of this mutation has been seen previously in ART-experienced PLWH in Pakistan (Shah et al., 2011) and when found in the ART-naive population, it may be present due to transmission from non-compliant ART-experienced individuals. The presence of DRMs, especially associated with resistance to zidovudine and nevirapine, two out of three that are drugs part of the ART regimen given to the children, may lead to ART failure (correlating with failure to suppress the viral load), and increase the possibility that these individuals may acquire severe form of disease (Gupta-Wright et al., 2020). A second DRM common in the outbreak sequences was the RT:K219N mutation, a thymidine analogue mutation associated with potential low-level resistance against zidovudine (Rhee et al., 2003). Presence of DRMs and spread of strains containing the mutation may lead to first line medications becoming obsolete and present challenges due to limited availability of second-line medications.

The strengths of the study are a relatively good sample size, active collection of samples during the outbreak and a comprehensive phylogenetic and phylodynamic analysis of the Larkana outbreak to identify subtype distribution and evolutionary relationship between sequences. The limitations include amplification of a single gene ( $p o l)$ only and short sequence length. While the parent case-control study showed that visits to both private and public sector health facilities and higher frequency of injections was associated with HIV-1 infection, we were unable to correlate the dyads and clusters with geographical data. The lack of samples of all biological mothers, where the mother was also HIV-positive, precluded investigation of the role of mother-to-child transmission. Future studies based on HIV-1 sequences sampled from different years (and HIV-1 risk groups) could shed light on the effectiveness of ART programs in Pakistan and provide an even more detailed picture of the Larkana outbreak.

In conclusion, our study findings showed that the Larkana paediatric outbreak did not originate from a single source and is likely a consequence of ongoing transmission within the high-risk groups of Larkana and introduced into the exposed individuals at risk of acquiring through poor infection prevention control practices in healthcare settings. Furthermore, the presence of multiple drug resistance mutations in the strains circulating in Larkana, especially to first-line ART drugs, is worrying as it limits treatment options. Large-scale transmission of resistant strains can hamper Pakistan's efforts to achieve the 90-90-90 goal (Maddali et al., 2016). These findings highlight not only the urgent need to improve blood safety and infection prevention control, but also the need for comprehensive molecular epidemiological studies and molecular surveillance to understand the distribution of different genotypes as well as origin, transmission, and drug resistance patterns.

\section{DATA AVAILABILITY STATEMENT}

The datasets presented in this study can be found in online repositories. The names of the repository/repositories and accession number(s) can be found below: https://www. ncbi.nlm.nih.gov/genbank/, MN698251, MN698252, MN698253, MN698255, MN698256, MN698257, MN698258, MN698259, MN698260, MN698261, MN698262, MN698263, MN698264, MN752136, MN752137, and MT748850-MT749178.

\section{ETHICS STATEMENT}

The studies involving human participants were reviewed and approved by Aga Khan University Ethics Review Committee (ERC \#2019-1536-4200). Written informed consent to participate in this study was provided by the participants' legal guardian/next of kin.

\section{AUTHOR CONTRIBUTIONS}

SA, RF, and FM conceived the study. SA, GN, DS, and WR performed the experiments. $S A$ and $G N$ wrote the first draft. SFM, AS, ShS, SM, SaS, SA, RF, PK, JE, and FM were involved in outbreak investigation. GN, DS, and AN cleaned the data and prepared the final datasheets. SA, GN, and DS have equal contributions in all experiments and data analysis. JE, RF, and FM had an equal contribution in project supervision. All authors contributed to the article and approved the submitted version.

\section{FUNDING}

This study was funded by the Higher Education Commission (grant no. 5217/Sindh/NRPU/R\&D/HEC/2016), Pakistan Science Foundation [grant no. PSF/Res/S-AKU/Med (488)], and World Health Organization (grant 2019/969219-0). RF was funded by the Wellcome Trust through a Senior Fellowship in Clinical Science (206316/Z/17/Z). GN acknowledges support from the Sub-Saharan African Network for TB/HIV-1 Research Excellence (SANTHE), a DELTAS Africa Initiative (grant \#DEL15-006). The DELTAS Africa Initiative was an independent funding scheme of the African Academy of Sciences (AAS)'s Alliance for Accelerating Excellence in Science in Africa (AESA) and supported by the New Partnership for Africa's Development Planning and Coordinating Agency (NEPAD Agency) with funding from the Wellcome Trust (grant \#107752/Z/15/Z) and the United Kingdom Government. 


\section{REFERENCES}

Abi-Habib, M., and Masood, S. H. I. V. (2019). Scandal Puts Focus on Pakistan's Health Care System. New York, NY: The New York Times.

Altaf, A. (2018). Delays and gaps in HIV programmes in Pakistan. Lancet HIV 5, e678-e679.

Altaf, A., Pasha, S., Vermund, S. H., and Shah, S. A. (2016). A second major HIV outbreak in Larkana, Pakistan. J. Pak. Med. Assoc. 66, 1510-1511.

Arif, F. (2019). HIV crisis in Sindh, Pakistan: the tip of the iceberg. Lancet Infect. Dis. 19, 695-696. doi: 10.1016/s1473-3099(19)30265-8

Baele, G., Lemey, P., Bedford, T., Rambaut, A., Suchard, M. A., and Alekseyenko, A. V. (2012). Improving the accuracy of demographic and molecular clock model comparison while accommodating phylogenetic uncertainty. Mol. Biol. Evolut. 29, 2157-2167. doi: 10.1093/molbev/ mss084

Baqi, S., Nabi, N., Hasan, S. N., Khan, A. J., Pasha, O., Kayani, N., et al. (1998). HIV antibody seroprevalence and associated risk factors in sex workers, drug users, and prisoners in Sindh, Pakistan. J. Acquir. Immune Deficien. Syndrom. Hum. Retrovirol. 18, 73-79. doi: 10.1097/00042560-19980501000011

Brenner, B. G., Roger, M., Routy, J. P., Moisi, D., Ntemgwa, M., Matte, C., et al. (2007). High rates of forward transmission events after acute/early HIV-1 infection. J. Infect. Dis. 195, 951-959. doi: 10.1086/512088

Chen, Y., Hora, B., DeMarco, T., Shah, S. A., Ahmed, M., Sanchez, A. M., et al. (2016). Fast dissemination of new HIV-1 CRF02/A1 recombinants in Pakistan. PLoS One 11:e0167839. doi: 10.1186/s12864-018-5380-8

Cholette, F., Joy, J., Pelcat, Y., Thompson, L. H., Pilon, R., Ho, J., et al. (2020). HIV1 phylodynamic analysis among people who inject drugs in Pakistan correlates with trends in illicit opioid trade. PLoS One 15:e0237560. doi: 10.1371/journal. pone. 0237560

Cotton, M. F., and Rabie, H. (2020). HIV outbreak in children in Pakistan: localised or more widespread? Lancet Infect. Dis. 20:269. doi: 10.1016/s1473-3099(19) 30746-7

Dawson, L., Benbow, N., Fletcher, F. E., Kassaye, S., Killelea, A., Latham, S. R., et al. (2020). Addressing Ethical Challenges in US-Based HIV Phylogenetic Research. J. Infect. Dis. 222, 1997-2006. doi: 10.1093/infdis/jiaa107

de Mendoza, C. (2019). UNAIDS Update Global HIV Numbers. AIDS Rev. 21, 170-171.

Debyser, Z., Van Wijngaerden, E., Van Laethem, K., Beuselinck, K., Reynders, M., De Clercq, E., et al. (1998). Failure to quantify viral load with two of the three commercial methods in a pregnant woman harboring an HIV type 1 subtype G strain. AIDS Res. Hum. Retroviruses 14, 453-459. doi: 10.1089/aid.1998. 14.453

Drummond, A. J., Rambaut, A., Shapiro, B., and Pybus, O. G. (2005). Bayesian coalescent inference of past population dynamics from molecular sequences. Mol. Biol. Evolut. 22, 1185-1192. doi: 10.1093/molbev/ msi103

Esbjörnsson, J., Mild, M., Audelin, A., Fonager, J., Skar, H., Bruun Jørgensen, L., et al. (2016). HIV-1 transmission between MSM and heterosexuals, and increasing proportions of circulating recombinant forms in the Nordic Countries. Virus Evolut. 2:vew010. doi: 10.1093/ve/vew010

Gill, M. S., Lemey, P., Faria, N. R., Rambaut, A., Shapiro, B., and Suchard, M. A. (2013). Improving Bayesian population dynamics inference: a coalescent-based model for multiple loci. Mol. Biol. Evolut. 30, 713-724. doi: 10.1093/molbev/ mss 265

Gill, M. S., Lemey, P., Suchard, M. A., Rambaut, A., and Baele, G. (2020). Online Bayesian Phylodynamic Inference in BEAST with Application to Epidemic Reconstruction. Mol. Biol. Evol. 37, 1832-1842. doi: 10.1093/molbev/ msaa047

Guindon, S., Dufayard, J.-F., Lefort, V., Anisimova, M., Hordijk, W., and Gascuel, O. (2010). New algorithms and methods to estimate maximum-likelihood phylogenies: assessing the performance of PhyML 3.0. Systemat. Biol. 59, 307-321. doi: 10.1093/sysbio/syq010

Gupta, S., Taylor, T., Patterson, A., Liang, B., Bullard, J., Sandstrom, P., et al. (2017). A Robust PCR Protocol for HIV Drug Resistance Testing on Low-Level Viremia Samples. Biomed. Res. Int. 2017:4979252.

Gupta-Wright, A., Fielding, K., van Oosterhout, J. J., Alufandika, M., Grint, D. J., Chimbayo, E., et al. (2020). Virological failure, HIV-1 drug resistance, and early mortality in adults admitted to hospital in Malawi: an observational cohort study. Lancet HIV 7, e620-e628.

Hasan, Z., Shah, S., Hasan, R., Rao, S., Ahmed, M., Stone, M., et al. (2018). Late diagnosis of human immunodeficiency virus infections in high-risk groups in Karachi, Pakistan. Int. J. STD AIDS 2018:956462418785264.

Hassan, A. S., Pybus, O. G., Sanders, E. J., Albert, J., and Esbjörnsson, J. (2017). Defining HIV-1 transmission clusters based on sequence data. AIDS 31:1211. doi: $10.1097 /$ qad.0000000000001470

Jeulin, H., Foissac, M., Boyer, L., Agrinier, N., Perrier, P., Kennel, A., et al. (2014). Real-life rilpivirine resistance and potential emergence of an E138A-positive HIV strain in north-eastern France. J. Antimicrob. Chemother. 69, 3095-3102. doi: 10.1093/jac/dku256

Khan, S., Zahid, M., Qureshi, M. A., Mughal, M. N., and Ujjan, I. D. (2018). HIV-1 genetic diversity, geographical linkages and antiretroviral drug resistance among individuals from Pakistan. Archiv. Virol. 163, 33-40. doi: 10.1007/ s00705-017-3564-1

Kosakovsky Pond, S. L., Weaver, S., Leigh Brown, A. J., and Wertheim, J. O. (2018). HIV-TRACE (TRAnsmission Cluster Engine): a Tool for Large Scale Molecular Epidemiology of HIV-1 and Other Rapidly Evolving Pathogens. Mol. Biol. Evol. 35, 1812-1819. doi: 10.1093/molbev/msy016

Kuiken, C., Korber, B., and Shafer, R. W. (2003). HIV sequence databases. AIDS Rev. 5, 52-61.

Larkin, M. A., Blackshields, G., Brown, N., Chenna, R., McGettigan, P. A., McWilliam, H., et al. (2007). Clustal W and Clustal X version 2.0. Bioinformatics 23, 2947-2948. doi: 10.1093/bioinformatics/btm404

Maddali, M. V., Gupta, A., and Shah, M. (2016). Epidemiological impact of achieving UNAIDS 90-90-90 targets for HIV care in India: a modelling study. BMJ Open 6:e011914. doi: 10.1136/bmjopen-2016-011914

Mir, F., Mahmood, F., Siddiqui, A. R., Baqi, S., Abidi, S. H., Kazi, A. M., et al. (2020a). HIV infection predominantly affecting children in Sindh, Pakistan, 2019: a cross-sectional study of an outbreak. Lancet Infect. Dis. 20, 362-370. doi: 10.1016/s1473-3099(19)30743-1

Mir, F., Nathwani, A. A., Simms, V., Abidi, S. H., Siddiqui, A. R., Hotwani, A., et al. (2020b). Investigation of an extensive outbreak of HIV infection among children in Sindh, Pakistan: a case-control study. Lancet HIV 10:e036723. doi: 10.1136/bmjopen-2019-036723

Nazziwa, J., Faria, N. R., Chaplin, B., Rawizza, H., Kanki, P., Dakum, P., et al. (2020). Characterisation of HIV-1 Molecular Epidemiology in Nigeria: Origin, Diversity, Demography and Geographic Spread. Sci. Rep. 10:3468.

Nduva, G. M., Hassan, A. S., Nazziwa, J., Graham, S. M., Esbjörnsson, J., and Sanders, E. J. (2020). HIV-1 Transmission Patterns Within and Between Risk Groups in Coastal Kenya. Sci. Rep. 10:6775.

Raees, M. A., Abidi, S. H., Ali, W., Khanani, M. R., and Ali, S. (2013). HIV among women and children in Pakistan. Trends Microbiol. 21, 213-214. doi: 10.1016/j.tim.2012.12.005

Rambaut, A., Lam, T. T., Max Carvalho, L., and Pybus, O. G. (2016). Exploring the temporal structure of heterochronous sequences using TempEst (formerly Path-O-Gen). Virus Evolut. 2:vew007. doi: 10.1093/ve/ vew007

Rhee, S. Y., Gonzales, M. J., Kantor, R., Betts, B. J., Ravela, J., and Shafer, R. W. (2003). Human immunodeficiency virus reverse transcriptase and protease sequence database. Nucleic Acids Res. 31, 298-303. doi: 10.1093/nar/ gkg100

Romero-Severson, E., Nasir, A., and Leitner, T. (2020). What Should Health Departments Do with HIV Sequence Data? Viruses 12:1018.

Shah, S., Xing, H., Altaf, A., Chen, B., Liao, L., Jia, Y., et al. (2011). Antiretroviral drug resistance mutations among treated and treatmentnaive patients in Pakistan: diversity of the HIV type 1 pol gene in Pakistan. AIDS Res. Hum. Retroviruses 27, 1277-1282. doi: 10.1089/aid.2010. 0324

Siddiqui, A. R., Ali Nathwani, A., Abidi, S. H., Mahmood, S. F., Azam, I., Sawani, S., et al. (2020). Investigation of an extensive outbreak of HIV infection among children in Sindh, Pakistan: protocol for a matched case-control study. BMJ Open 10:e036723. doi: 10.1136/bmjopen-2019036723

Suchard, M. A., Lemey, P., Baele, G., Ayres, D. L., Drummond, A. J., and Rambaut, A. (2018). Bayesian phylogenetic and phylodynamic data 
integration using BEAST 1.10. Virus Evolut. 4:vey016. doi: 10.1093/ve/ vey016

Tariq, U., Parveen, A., Akhtar, F., Mahmood, F., Ali, S., and Abidi, S. H. (2018). Emergence of circulating recombinant form 56_cpx in Pakistan. AIDS Res. Hum. Retroviruses 34, 1002-1004. doi: 10.1089/aid.2018. 0128

Taylor, B. S., Sobieszczyk, M. E., McCutchan, F. E., and Hammer, S. M. (2008). The challenge of HIV-1 subtype diversity. N. Engl. J. Med. 358, 1590-1602. doi: 10.1056/nejmra0706737

van Zyl, G., Bale, M. J., and Kearney, M. F. (2018). HIV evolution and diversity in ART-treated patients. Retrovirology 15:14.

Visco-Comandini, U., Cappiello, G., Liuzzi, G., Tozzi, V., Anzidei, G., Abbate, I., et al. (2002). Monophyletic HIV type 1 CRF02-AG in a nosocomial outbreak in Benghazi, Libya. AIDS Res. Hum. Retroviruses 18, 727-732. doi: 10.1089/ 088922202760072366

Waheed, Y., and Waheed, H. (2017). Pakistan needs to speed up its human immunodeficiency virus control strategy to achieve targets in fast-track acquired immune deficiency syndrome response. World J. Virol. 6, 46-48. doi: 10.5501/wjv.v6.i2.46

Weinberg, J. L., and Kovarik, C. L. (2010). The WHO Clinical Staging System for HIV/AIDS. Virtual Mentor. 12, 202-206. doi: 10.1001/virtualmentor.2010.12.3. cprl1-1003
Wensing, A. M., Calvez, V., Ceccherini-Silberstein, F., Charpentier, C., Günthard, H. F., Paredes, R., et al. (2019). 2019 update of the drug resistance mutations in HIV-1. Topics Antiviral Med. 27:111.

Conflict of Interest: The authors declare that the research was conducted in the absence of any commercial or financial relationships that could be construed as a potential conflict of interest.

Publisher's Note: All claims expressed in this article are solely those of the authors and do not necessarily represent those of their affiliated organizations, or those of the publisher, the editors and the reviewers. Any product that may be evaluated in this article, or claim that may be made by its manufacturer, is not guaranteed or endorsed by the publisher.

Copyright (C) 2021 Abidi, Nduva, Siddiqui, Rafaqat, Mahmood, Siddiqui, Nathwani, Hotwani, Shah, Memon, Sheikh, Khan, Esbjörnsson, Ferrand and Mir. This is an open-access article distributed under the terms of the Creative Commons Attribution License (CC BY). The use, distribution or reproduction in other forums is permitted, provided the original author(s) and the copyright owner(s) are credited and that the original publication in this journal is cited, in accordance with accepted academic practice. No use, distribution or reproduction is permitted which does not comply with these terms. 